\title{
GENETICAL EVIDENCE ON ALTERNATIVE MATERNAL AND MENDELIAN HEREDITARY ELEMENTS IN PETUNIA HYBRIDA
}

\author{
RAFAEL FRANKEL \\ The Volcani Institute of Agricultural Research, Bet Dagan, Israel
}

Received 2.iv.70

\section{INTRODUCTION}

Extrachromosomal hereditary factors controlling male sterility in higher plants are prominent in the plant kingdom and have practical and potential applications in hybrid seed production. Cytoplasmic male sterility has been studied intensively for a long time (see reviews of Edwardson, 1956; Duvick, 1959; Jain, 1959), yet the body of experimenal evidence produced still does not give critical indications on the nature as well as the extrachromosomal site or carrier of the sterility factor (Edwardson, 1968).

Although much speculation can be made as to the carriers of the extrachromosomal genetic information (Rhoades, 1950; Edwardson and Corbett, 1961 ; Atanasoff, 1964; Michaelis, 1964), no association between the altered function in microsporogenesis and presence, absence, or altered morphology of cytoplasmic organelles, constituents, or inclusions, has yet been conclusively demonstrated. Evidence from some graft experiments with Petunia (Frankel, 1956, 1962; Edwardson and Corbett, 1961; Bianchi, 1963), beet (Curtis, 1967) and corn (Borovskij, 1960), but not yet with other plants (tobacco-Sand, 1960; Burk, 1960; pepper-Ohta, 1961; wheat-Lacadena, 1968; Epilobium-Michaelis, 1940), suggested asexual transformation to male sterility in the progeny of the cytofertile symbiont of heteroplastic grafts.

Responsibility for such asexual transformation has been attributed to the presence of and genetic information of latent viruses in cytoplasmic malesterile plants (Edwardson and Corbett, 1961; Atanasoff, 1964). This type of interpretation must assume reproduction and transmission of such viruses to be kept in pace with the measure of cell multiplication and transmission of cytoplasmic organelles in soma and germ line. Hence, distinction between viral and host cell hereditary information cannot be based on the mode of reproduction and transmission of the hereditary element. A distinction between host cell and infective agents hereditary information based on criteria such as infectivity and mobility also seems to be inadequate, if we consider mechanisms of sexual and asexual transmission of hereditary material or information (processes like fertilisation, transformation, and even non-autonomous behaviour of symbionts). Thus, in the absence of other relevant criteria, such distinctions and definitions became a matter of semantics or convention.

Evidence given in this paper suggests that we rather could find refuge in the apparent link which can be established in bacteria between epinuclear hereditary elements and pathological agents on the one hand and the chromosomal complement on the other hand, as exemplified by the episomal scheme (Jacob and Wollman, 1961). Evidence available now suggests strongly that "genetic interactions which may result in the insertion into or expulsion from the chromosome of DNA segments occur as a natural 
property of the genetic material-at least in the form in which it exists in bacterial cells" (Hayes, 1969). The body of genetical evidence given here supports the notion of alternative maternal and Mendelian hereditary elements for male sterility in Petunia hybrida proposed earlier (Frankel, 1963; 1967).

\section{Materials AND MEthods}

The initial evidence on the induction of male sterility in part of the progeny of fertile petunia symbionts grafted with cytoplasmic male-sterile symbionts involved 15 reciprocal grafts and was based on two fertile symbionts yielding male-sterile plants (Frankel, 1956). Confirmation of such induction was found in a second series of graft experiments involving 10 fertile and 10 male-sterile graft components (Frankel, 1962, 1964).

The present paper is based mainly on a comprehensive genetic analysis of the progeny of two male-fertile graft symbionts from the above-mentioned second series of experiments-one including cytoplasmic and the other monogenic recessive Mendelian male-sterile offsprings. Since full details on material and experimental procedures have been given previously (Frankel, 1962, 1964), a general outline of these will suffice here.

Genetic background of both male-sterile and male-fertile material was essentially of the diploid petunia cultivar "Rosy Morn". Isogenic material, produced by clonal propagation, was used as graft symbionts and control plants. Various arrangements of graft unions (mainly in cleft and approachgraft techniques) were used to study the transmission phenomenon. Fertile and male-sterile components were used as stocks, as scions, and as interscions in heteroplastic sandwich grafts. Ungrafted clones served as check plants. Glones were made also from the graft symbionts.

\section{RESULTS}

\section{Recapitulation of main features of grafting experiments}

Although fertile symbionts grafted with male-sterile symbionts remained autonomous in the expression of male fertility, male-sterile plants appeared in four cases in the progeny of the fertile symbionts. All four cases occurred in the progeny of symbionts grafted during the summer months. In the progeny of the ungrafted donor plants serving as controls, no male steriles were ever found (Frankel, 1964). A summarised pedigree of one of the cases of graft-induced transmission of male sterility to the progeny of a fertile graft component is given in fig. 1 .

The main features of the grafting experiments can be outlined as follows:

\section{(a) Universal and persistent phenotypic autonomy of the graft symbionts}

As mentioned previously (Frankel, 1962), parallel expression of male fertility in the graft symbionts and their ungrafted donor clone was general. Fertiles produced abundant functional pollen and steriles no pollen at all for the lifetime of the components; this remained the case also when the progeny of the fertile graft component showed male sterility at the same time. Graft components were maintained for up to five years.

The stability of the autonomy indicates that no simple adaptation of the element responsible for sterility could account for the autonomy and for 
certain ratios of fertile and sterile $\mathrm{F}_{1}$ progeny. However, the possibility that certain genotypes might not behave autonomously, or that the induction of male sterility takes place only after sporogenesis, can not yet be ruled out.

\section{(b) Multidirectional transmission of male sterility between graft components}

In cases where male sterility was realised in the progeny of a male-fertile graft symbiont, realisation was uniform in the progeny of clonal components of different types (such as stocks, scions, interscions, etc.), in heteroplastic grafts (Frankel, 1965). Specifically, this means upward, sideways and downward transmission or effect of the sterility-determining entity.

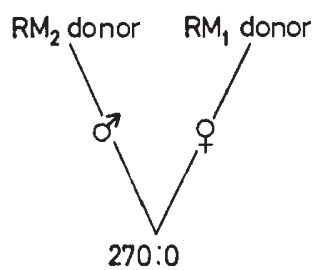

$270: 0$

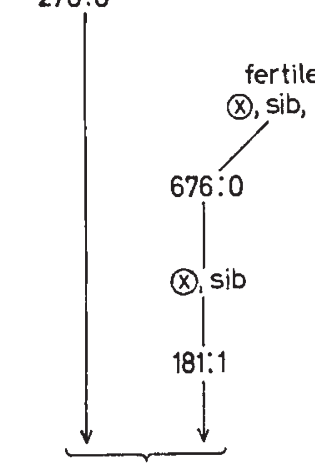

all fertiles to $F_{9}$

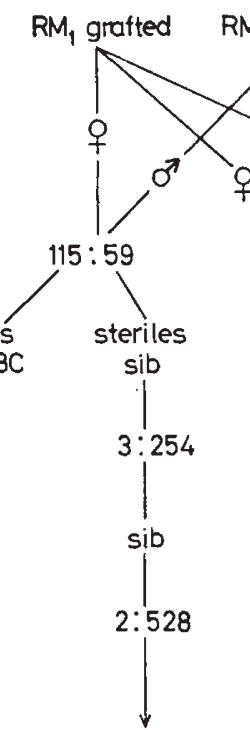

all steriles to $F_{9}$ $\mathrm{RM}_{2}$ grafted $\quad \mathrm{RM}_{2}$ detached $\mathrm{RM}_{1}$ detached

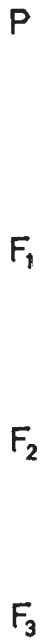

Fig. 1.- Summarised partial pedigree of $\mathrm{RM}_{1}$ : the normal case.

$\mathrm{RM}_{1}$ donor = ungrafted clone of a fertile plant of " Rosy Morn".

$\mathrm{RM}_{2}$ donor = ungrafted clone of a fertile plant of "Rosy Morn".

$\mathrm{RM}_{1}$-grafted = fertile " Rosy Morn " graft symbiont with cytoplasmic male sterile.

$\mathrm{RM}_{2}$-grafted = fertile " Rosy Morn" graft symbiont with cytoplasmic male sterile.

$\mathrm{RM}_{1}$-detached = rooted cutting detached from $\mathrm{RM}_{1}$-grafted.

$\mathrm{RM}_{2}$-detached = rooted cutting detached from $\mathrm{RM}_{2}$-grafted.

Ratios are given-male fertiles: male steriles.

(c) Lack of time effects on the frequency of occurrence of male-sterile individuals in the progeny of fertile graft symbionts

Proportions of male-sterile and fertile plants in the progeny of fertile graft symbionts (all grafted under high-temperature regime) were independent of the age of the graft union (time lapse between grafting date and gamete production up to five years) and of the sequence of individual flowers and their distance from the graft union, provided that flowers were at stages before gametogenesis at the time of grafting. Furthermore, numerical proportions of male-sterile and fertile plants in the progeny of fertile graft symbionts were independent of the age and planting season of the seed. Hence, no decrease in the asexual transmission of the agent responsible for male sterility is evident with increasing age of the seed. 
(d) Genotype of fertile graft symbiont and pollen parent controls the transmission of the cytoplasmic factor to its progeny

Studies with some mother symbionts pollinated by different pollen sources (see also fig. 1), or with different mother symbionts pollinated by the same pollen, indicate genotypic control of the asexual transmission to progeny of the cytoplasmic male sterility factor. Furthermore, only part of the fertile graft components produced $\mathrm{F}_{1}$ progeny including male-sterile plants (Frankel, 1962, 1964).

\section{(e) Maternal inheritance of male sterility induced in the progeny of fertile graft symbionts}

Male-sterile plants appearing in the progeny of fertile graft symbionts pollinated with any pollen gave only male-sterile offspring for nine generations. Thus, the induced male-sterile trait behaved identically to the trait found in the original male-sterile graft symbiont (fig. 1).

(f) Dependence of induction of male sterility in the progeny of the fertile graft symbionts on male as well as female gametes

Induction could be realised only if both pollen and egg donor had been in an active graft union with a male-sterile component at the time of pollination. This has been indicated both by progeny resulting from comparative pollination using grafted plants, ungrafted donor plants, and cuttings made from grafted symbionts. The induction, though realised very shortly after grafting, appears to be lost immediately after a clone is detached from the graft union.

Extensive investigations with clones of three fertile genotypes from which we recovered male sterility in some of the progeny of the fertile graft symbiont, and used in repeated graft experiments, all indicated the above features exemplified by the pedigree of $\mathrm{RM}_{1}$ (fig. 1), that is :

instant response after grafting;

requirement that both gametes are produced on a grafted symbiont;

instant loss of induction after detachment from the graft of the ovule or pollen parent; and

the trait induced exhibits cytoplasmic inheritance similar to that in the male-sterile graft symbiont.

\section{Inheritance of male sterility in the progeny of clone $R M_{5}$}

One fertile genotype, $\mathrm{RM}_{5}$, from which male-sterile plants in the progeny of fertile graft symbionts were recovered, and which was used in repeated graft experiments, exhibited features different from the three genotypes mentioned above (details in Frankel, 1962, and unpublished). Figure 2 gives a summarised pedigree of the progeny of clone $\mathrm{RM}_{5}$.

In the exceptional case of clone $\mathrm{RM}_{5}$ the progeny from selfing, all fertile symbionts grafted with male-sterile symbionts, showed the induction of Mendelian male sterility, that is, a mode of inheritance different from the type of cytoplasmically inherited male sterility present in the male-sterile graft symbiont. Furthermore, using pollen from ungrafted plants in pollinating the graft symbiont (that is, pollen from the ungrafted clone-the donor, or other ungrafted plants) verified the Mendelian inheritance pattern in the 
progeny. The data indicate that one recessive gene was responsible for the sterility trait in the progeny of the graft symbiont. The original fertile graft symbionts $\mathrm{RM}_{5}$ appeared to be heterozygous for the responsible locus, since back-crosses of the sterile progeny to the symbiont gave a $1: 1$ ratio.

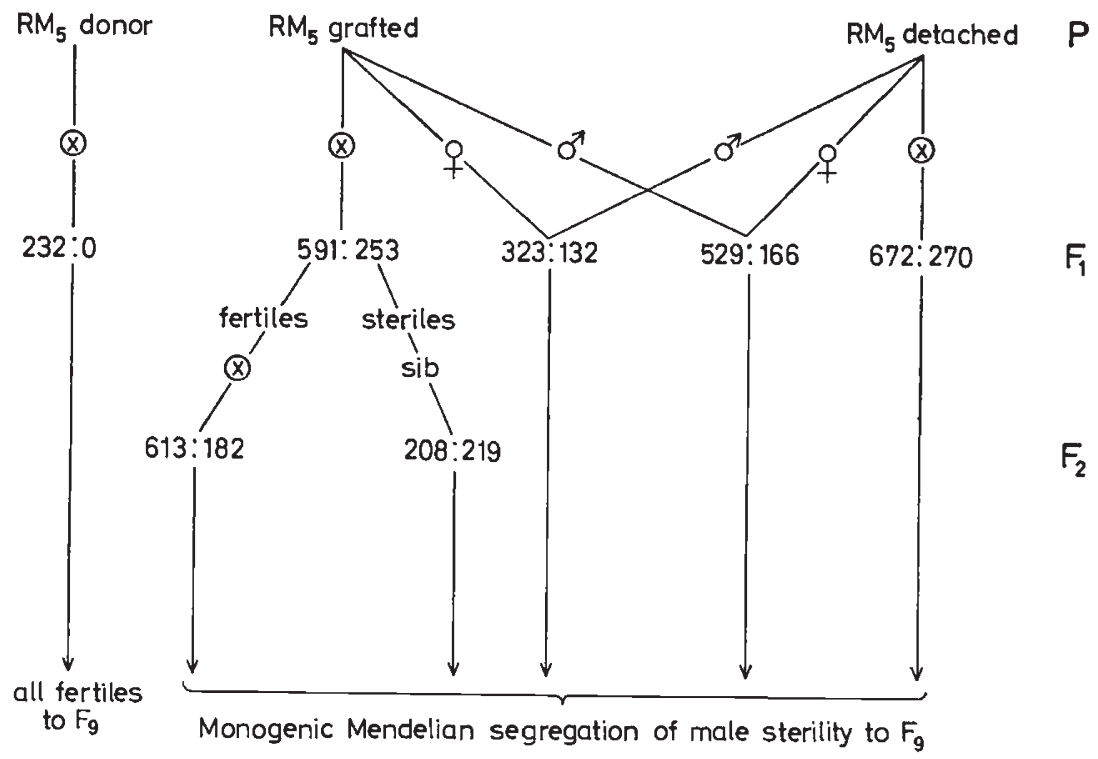

FIG. 2.-Summarised partial pedigree of $\mathrm{RM}_{5}$ : the exceptional case.

$\mathrm{RM}_{5}$ donor = ungrafted clone of fertile plant of "Rosy Morn".

$\mathrm{RM}_{5}$-grafted = fertile "Rosy Morn" graft symbiont with male sterile.

$\mathrm{RM}_{5}$-detached = Rooted cutting detached from $\mathrm{RM}_{5}$-grafted.

Ratios are given-male fertiles : male steriles.

Another difference from the normal case found with clone $\mathrm{RM}_{5}$ was the latency of the induction of male sterility even after detachment of the ovule parent, pollen parent or both from the graft union. In other words, induction of Mendelian male sterility was also realised when the grafted symbiont was pollinated with pollen of the detached symbiont. Selfing the cutting taken from the fertile graft symbiont resulted in progeny exhibiting Mendelian male sterility, regardless of the time lapse between separation of the cutting and pollination.

\section{Relationship between the two types of induced male sterility}

In order to establish a possible relationship between the two types of induced male sterility, reciprocal crosses were made between parents of the two progenies (graft symbionts $\mathrm{RM}_{1}$ and $\mathrm{RM}_{5}$ ). Both reciprocal crosses yielded only fertile progeny in the first generation.

Since cytoplasmic male sterility originated in progenies of the fertile graft symbiont $\mathrm{RM}_{1}$ when grafted, further generations of the cross $\mathrm{RM}_{\mathrm{L}^{-}}$ grafted (as the female parent) $\times \mathrm{RM}_{5}$-grafted were grown. Results of the second and third generation of this cross show clearly the induction of Mendelian male sterility in spite of the maternal cytoplasm coming from plant $\mathrm{RM}_{1}$-grafted. Figure 3 shows the pedigree and data of progeny 
resulting from a cross between the two graft symbionts $R M_{1}$ and $R M_{5}$, that is, a cross in the "induction phase".

The next step was to determine whether chromosomal integration means the establishment of a male sterility (or fertility) gene, and whether this gene is really an alternative to the cytoplasmic factor, rather than plasmon-sensitive and fertility-restorer alleles. The test was based on crosses between induced and original cytoplasmic male-sterile females and heterozygous male fertiles $E \boldsymbol{e}$. It is obvious that the cytoplasmic male-sterile line could not be used as male parent in a reciptocal cross, but fertiles originating from $\mathrm{RM}_{1}$ grafted were used in crosses as males and showed consistent genic male sterility in the progeny.

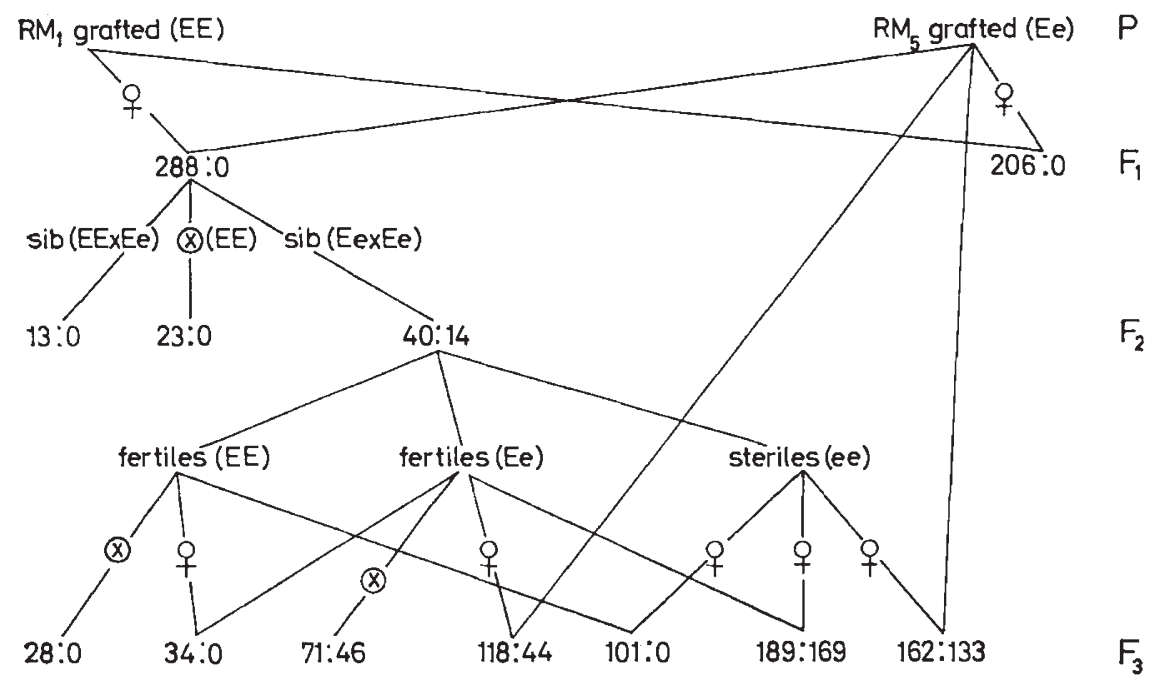

FIG. 3.-Pedigree of intercross between graft symbionts $R M_{1}$ and $R M_{6}$ (cross in the induction phase). Notation as in figs. 1 and 2.

The following crosses were made to study the interaction between the two types of induced male sterility and the original type of cytoplasmic male sterility present in the male-sterile graft symbiont:

1. Induced cytoplasmic male-sterile female $\times$ heterozygous male-sterile male $(E e)$.

2. Induced cytoplasmic male-sterile female $\times$ homozygous fertile male $(E E)$.

3. Original cytoplasmic male-sterile female $\times$ heterozygous male-sterile male $(E e)$.

4. Original cytoplasmic male-sterile female $\times$ homozygous fertile male $(E E)$.

Only cytoplasmic male-sterile progeny were obtained in all these crosses. This obviously means that the nuclear gene for fertility did not have a restoring effect on the cytoplasmic male sterility.

Figure 4 gives the pedigree of crosses between induced and original cytoplasmic male-sterile females and homozygous or heterozygous genic male fertiles. We refer to these crosses as being in the "combining phase", as opposed to the "induction phase". 


\section{Discussion}

Interpretation of the experimental results presented may be based on a working hypothesis of alternative hereditary elements for male sterility in petunia. Results of the induction of male sterility in $\mathrm{F}_{1}$ of clonal components as outlined in subsections 3.1 and 3.2 are summarised in table 1 .

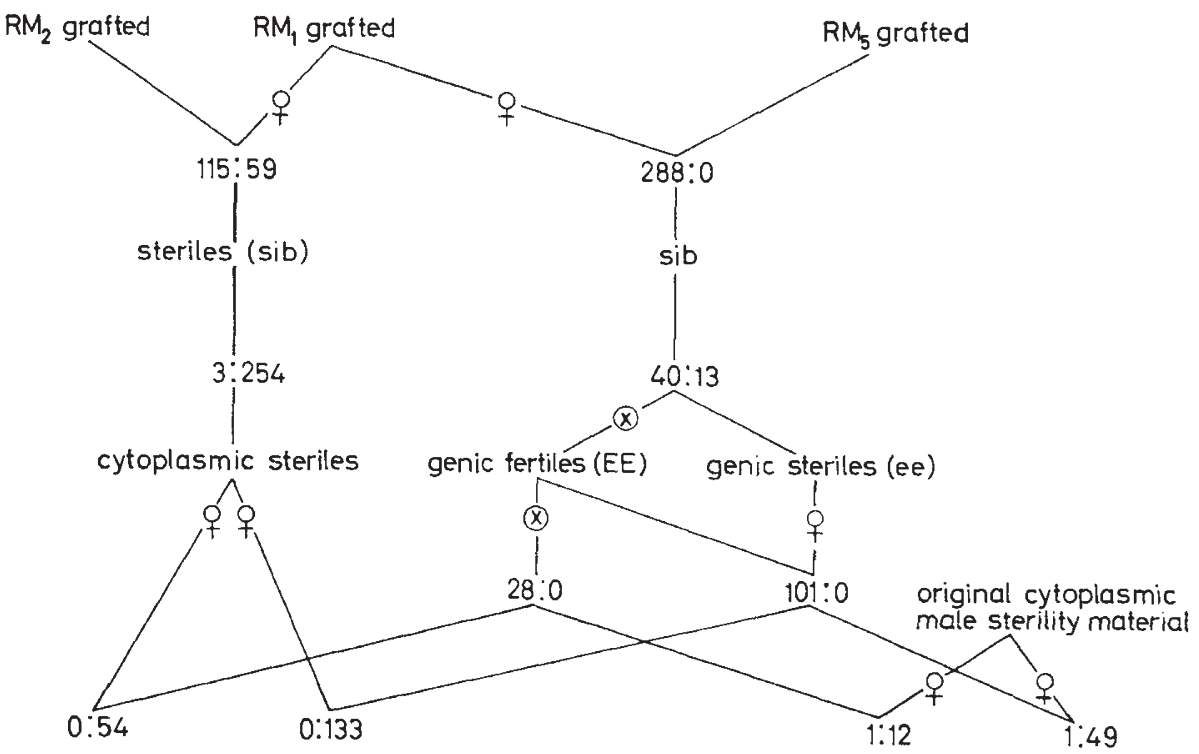

Frg. 4.--Pedigree of crosses between induced cytoplasmic male steriles and heterozygous male fertiles (cross in the combining phase).

The inference from table 1 and from additional evidence presented in the previous sections brings us to the following conclusions:

(a) Two types of male sterility have been induced in the progeny of two different fertile graft symbionts:

1. the cross $R M_{1}$-grafted $\times R_{2} M_{2}$-grafted yielded progeny exhibiting maternal inheritance of male sterility;

2. selfing $\mathrm{RM}_{5}$-grafted yielded progeny exhibiting Mendelian inheritance of male sterility (one recessive factor).

(b) Both parents seem to require active graft connections with a malesterile symbiont at the time of pollination in order to realise the transmission phenomenon of cytoplasmic male sterility in the progeny of $\mathrm{RM}_{\mathbf{1}}$-grafted.

(c) Latency of the transmission phenomenon was shown in cuttings of plant $\mathrm{RM}_{5}\left(\mathrm{RM}_{5}\right.$-detached) and Mendelian inheritance of male sterility was realised in the progeny of the cuttings or of the graft symbiont pollinated with the pollen of the cutting $\left(\mathrm{RM}_{5}\right.$-grafted $\times \mathrm{RM}_{5}$-detached). In contrast to this, induction of male sterility was lost in $\mathrm{RM}_{1}$-detached and all progeny were fertile, whereas cytoplasmic male sterility was realised in the progeny of the grafted symbiont $\mathrm{RM}_{1}$-grafted.

It has to be emphasised at this point that we have to distinguish between the genotypic control of the induction of male sterility and its inheritance pattern after induction has been achieved. 
In the normal case, genotypic control of the induction has been indicated through both gametes in the induction phase (the time when the symbiont is in active graft union). Nevertheless, no restorer genes are found for the trait induced in the progeny. Genes controlling the transmission or induction do not restore the sterility induced in the progeny. This is clearly indicated by backcrosses to the fertile graft symbiont.

In the exceptional case, genotypic control has been indicated through either of the gametes in the induction phase. The inheritance pattern of the fertile and male-sterile progeny suggests that the allele involved in the induction phase behaves like an activated male-sterility allele. It seems that the original graft symbiont $\mathrm{RM}_{5}$ was heterozygous for the particular locus $(E e)$. This is actually shown by the back-cross progeny giving a $1: 1$ ratio with steriles, and a $3: 1$ or $4: 0$ ratio with fertiles.

TABLE 1

Induction of male sterility in $F_{1}$ clonal components*

Clonal components used in selfing

Grafted $\times$ grafted

Grafted $\times$ ungrafted

Ungrafted $\times$ grafted

Grafted $\times$ detached

Detached $\times$ grafted

Detached $\times$ detached

Donor-selfed

Inheritance pattern after induction in $\mathrm{F}_{1}$

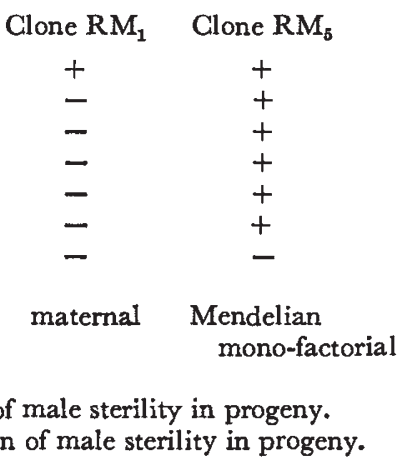

The question might be posed-How do we know whether the Mendelian male sterility resulted from the graft, or from a spontaneous somatic mutation? There are a number of reasons for believing that the Mendelian male sterility was actually a result of the graft, although the evidence is not entirely critical. It seems very unlikely that genic male sterility of a type unknown in Petunia should appear as a somatic mutation just in the graft symbiont affecting all cells giving rise to sporogeneous cells. We have maintained the graft symbiont for five years and taken repeated cuttings from it and also propagated repeatedly the cuttings-but all sexual progeny showed Mendelian male sterility. On the other hand, the donor plant, being an identical genotype originally, gave only fertile progeny.

We therefore prefer to relate both types of male sterility induced in the progeny of the fertile graft symbionts to the cytoplasmic factor present in the cytoplasmic male-sterile symbiont. Such a possible relation raises the question of whether the two types of male sterilities are alternative hereditary elements, perhaps analogous to the episomal elements in bacteria. In such an analogy the cytoplasmic male sterility would represent the autonomous state and the Mendelian male sterility the integrated state.

A graphical interpretation of the normal and exceptional cases under our working hypothesis is given in figs. 5 and 6 , respectively. 
We will consider now two critical tests for the proposition of alternative hereditary elements in the two cases of male sterility we have discussed:

(a) We have to show the identity or direct relation between the elements in the sterile graft component and the elements found in the progeny of the male-fertile graft component. The induction of both types of male sterility in the progeny of the fertile graft symbionts and not in the progeny of

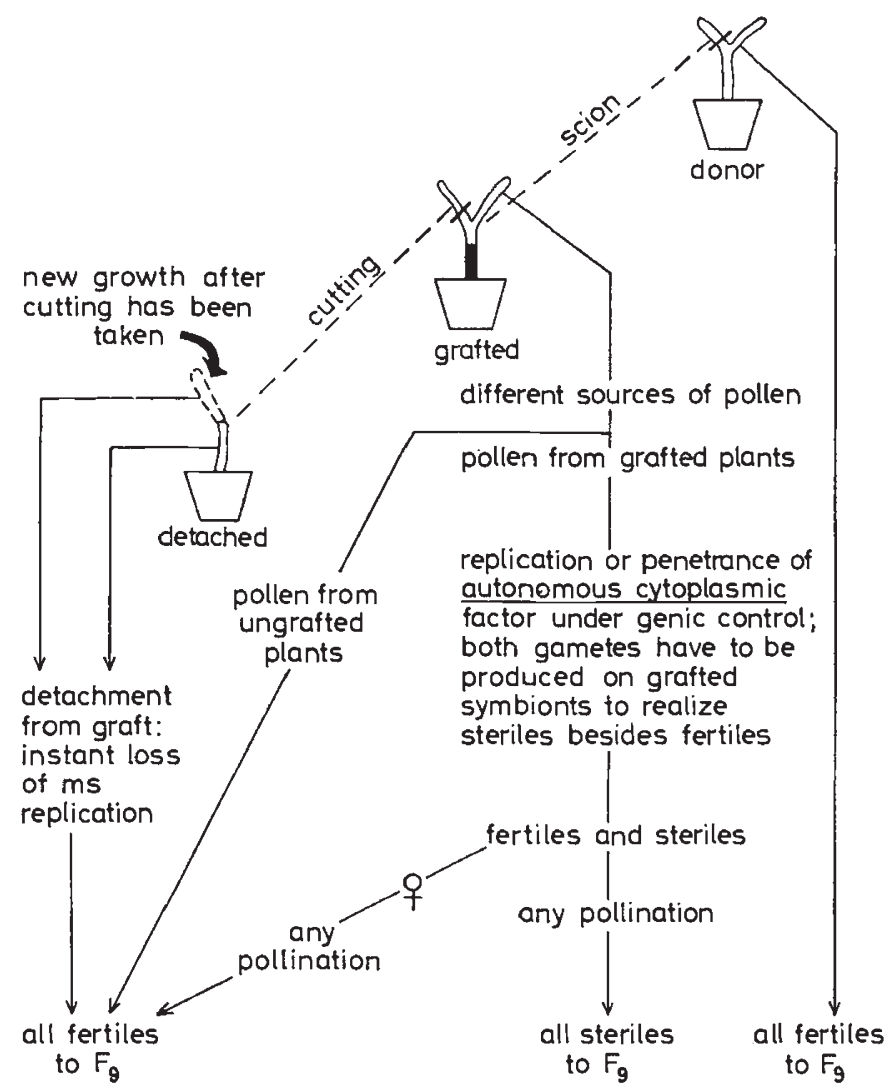

FIG. 5.-Interpretation of induction of male sterility ( $\mathrm{ms}$ ) in clone $\mathrm{RM}_{1}$ : the normal case.

control plants (i.e. donor plants) strongly suggests such a relation. In the case of the cytoplasmic factor found after induction, its inheritance, stability, and absence of restorer genes indicate the identity of the induced and the original factor.

(b) We have to establish the relationship between the two types of induced male sterility. In other words, we have to demonstrate that these are hereditary alternatives, and if analogous to episomes they should also be mutually exclusive. Results of the reciprocal crosses made between parents of the two progenies (crosses in the induction phasè-see fig. 3) show that in the phase of the active graft union the genic type of male sterility prevails. Our interpretation is that a chromosomal integration takes place in the particular genotype of the $\mathrm{RM}_{5}$ symbiont and an exclusion occurs of the cytoplasmic factor in the $\mathrm{RM}_{1}$ cytoplasm. This interpretation is shown 
graphically in fig. 7. Crosses between induced (and original) cytoplasmic male sterile females and homozygous $(E E)$ or heterozygous $(E e)$ male fertiles from the $\mathrm{RM}_{5}$ progeny (crosses in the combining phase-see fig. 4) indicate on the other hand absence of restoration of cytoplasmic male sterility by fertility alleles.

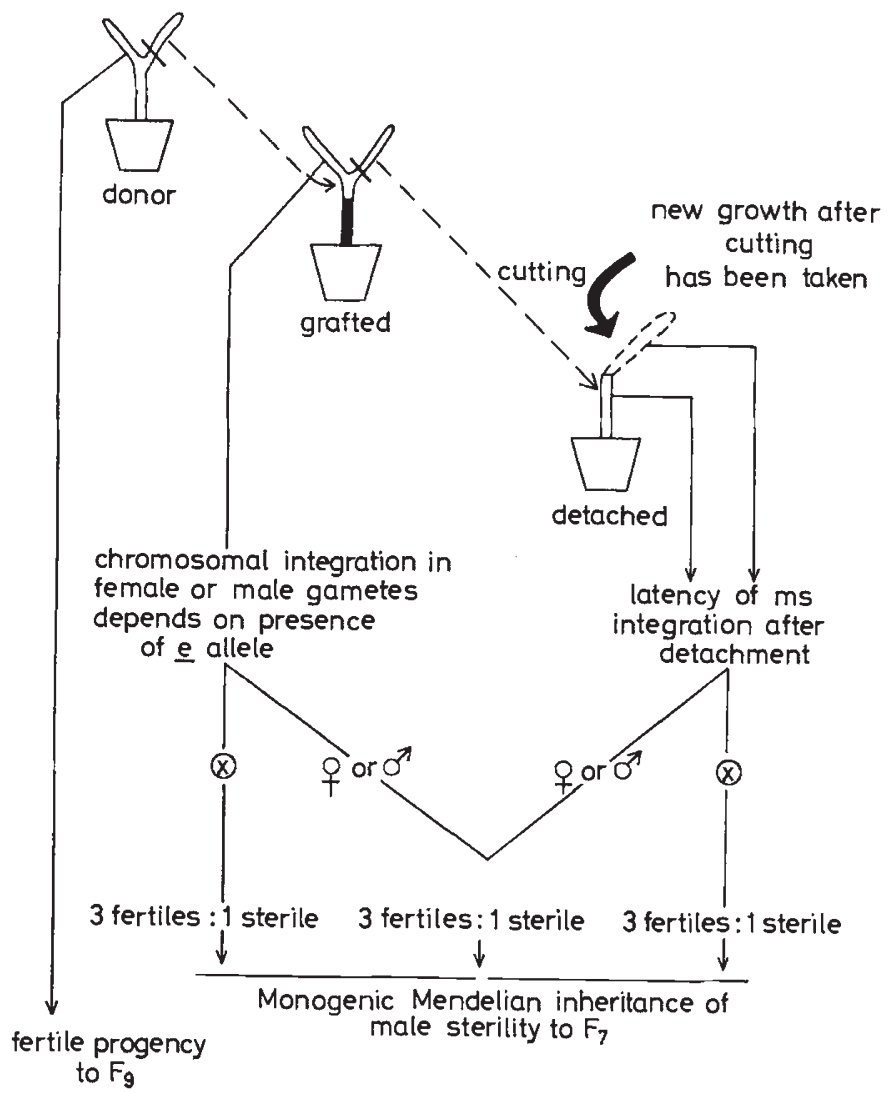

Fig. 6.-Interpretation of induction of male sterility $(\mathrm{ms})$ in clone $\mathrm{RM}_{b}\left(E_{e}\right)$ : the exceptional case.

We may thus summarise the results of crosses in the " induction phase" and the "combining phase" as follows:

1. Reciprocal crosses between $R_{1}$-grafted and $R_{1} M_{5}$-grafted showed the prevalence of the Mendelian factor over the cytoplasmic factor of male sterility in the graft generation ("induction phase").

2. On the other hand, no restoring effect by the monofactoral Mendelian factor (induced in the progeny of $\mathrm{RM}_{5}$-grafted) was found affecting cytoplasmic male sterility.

It appears, therefore, that only one type of hereditary factor exists at one time, that the second type is excluded by the presence of the first, and that such exclusion is mutual.

The prevalence of the genetic male sterility during the induction phase may be explained as follows: Recalling that cytoplasmic male sterility is 
induced only if both gametes carry the cytoplasmic element, the sporophyte carrying the "activated" (integrated) $e$ allele can not induce cytoplasmic male sterility; this is so because of mutual exclusiveness: such a sporophyte will not carry the episomal element. On the other hand, it should be expected that the episomal element, not being restored by the fertility allele, will prevail during the combining phase. The presence of such a fertility allele has to be assumed in the original fertile material.

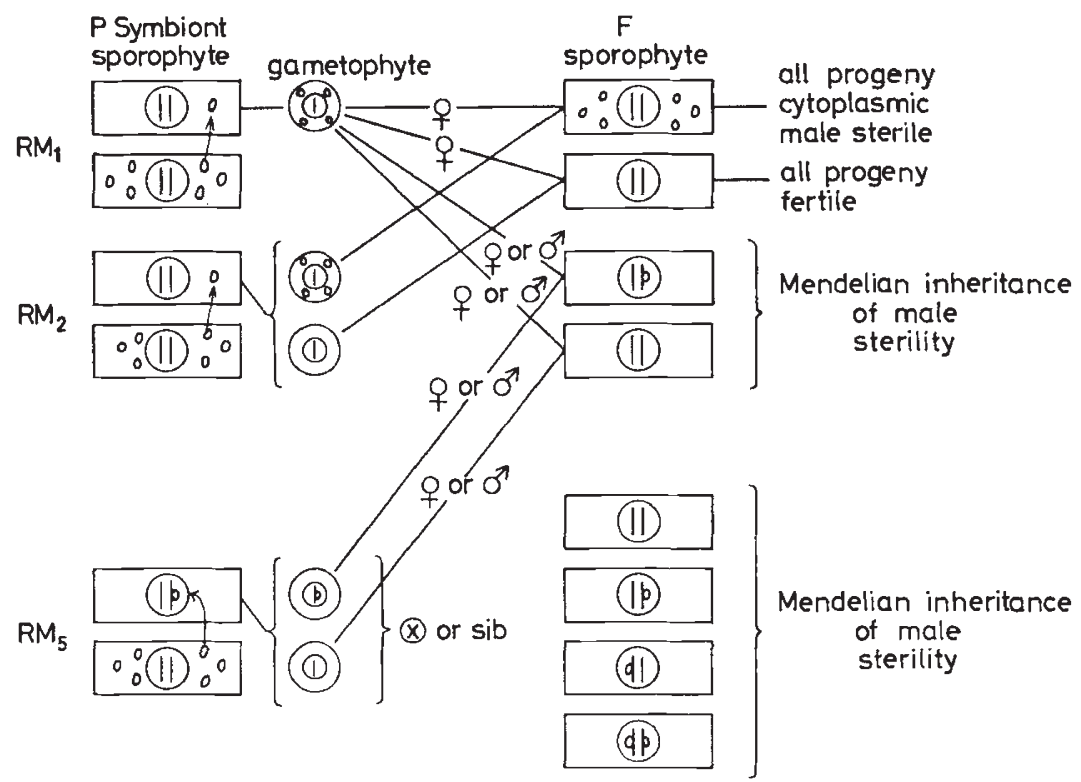

FIG. 7.-Interpretation of crossing results in the induction phase.

It is a pity that we are not able to self the progeny of the combining phase to see if actually the integration of the $e$ allele has been reversed, that is, excluded by the cytoplasmic factor. Current work in pollinating the cytoplasmic male-sterile progeny of the combining phase for more generations with $E, E$ and $E$, $e$ pollen still continues to show maternal inheritance.

Finally we should, however, keep in mind, that although we have strong indications that the genic male-sterility factor has in effect been produced by the cytoplasmic male-sterility factor, we still lack the critical proof for our hypothesis of genetical alternatives for male sterility in Petunia. Our working hypothesis has so far mainly been based on the apparent mutual exclusion of the two types of hereditary elements. We have not, as yet, produced the " autonomous state" from the " integrated state". In other words, although we seem to have induced genic male sterility from cytoplasmic male sterility, we have not done the opposite. Future work will have to provide the final critical proof required for our hypothesis of alternative maternal and Mendelian hereditary elements for male sterility in Petunia.

\section{Summary}

1. Genetic analysis of male sterility induced in the progeny of fertile graft components grafted with cytoplasmic male-sterile components of 
Petunia showed induction of two types of male sterility: one exhibiting inheritance, as did the original trait in the male-sterile graft component; the other exhibiting monogenic recessive Mendelian male sterility.

2 . Both gametes have to be produced on symbionts grafted with malesterile components to realise induction of cytoplasmic male sterility in the progeny of the fertile graft component. The induction of genic male sterility was found with either one of the gametes produced on a fertile symbiont grafted with a male-sterile component.

3. Latency of the induction phenomenon was found in a cutting of a graft symbiont giving genic male-sterile progeny. In contrast to this, induction was lost in a cutting of a graft symbiont giving cytoplasmic male-sterile progeny.

4. In the "induction phase" (graft generation) the genic type of male sterility, if induced, prevails. On the other hand, no restoring effect on the cytoplasmic trait by the monogenic sterility factor has been found in later generations.

5. The genetical evidence indicates asexual transmission of a cytoplasmic factor in Petunia, chromosomal integration of a cytoplasmic factor in a higher plant, and mutual exclusive cytoplasmic and chromosomal genetic alternatives for male sterility in Petunia.

Acknowledgments.-Contribution from the Volcani Institute of Agricultural Research, Bet Dagan, Israel, 1970 Series, No. 1681-E, supported in part by grant FG-IS-171 of the United States Department of Agriculture. Authorised by U.S. Public Law 480.

Thanks are due to Mrs. Gilboa for her excellent technical assistance.

\section{REFERENGES}

AtANASOFF, D. 1964. Viruses and cytoplasmic heredity. Z. Pfianzenzücht., 51, 197-214. bianchi, F. 1963. Transmission of male sterility in Petunia by grafting. Genen en Phaenen, $8,36-43$.

BOROVSKI, M. I. 1960. The transfer of the character of male sterility in maize by vegetative hybridisation. (Russian) Proc. Darwin Fubil. Conf. Kisinev, 209, 212.

BURK, L. G. 1960. Male sterile flower anomalies in interspecific tobacco hybrids. F. Hered., $51,27-29$

GURTIS, G. J. 1967. Graft-transmission of male sterility in sugar beet (Beta vulgaris L.). Euphytica, 16, 419-424.

Duvick, D. N. 1959. The use of cytoplasmic male sterility in hybrid seed production. Econ. Bot., 13, 167-195.

EDWARDSON, J. R. 1956. Cytoplasmic male sterility. Bot. Rev., 22, 696-738.

EDWARDson, J. R. 1968. The nature of cytoplasmic factors inducing male sterility. Proc. XII Int. Congr. Genetics, 2, 226-227.

EDWARDSON, J. R., AND CORBETT, M. K. (1961). Asexual transmission of cytoplasmic male sterility. Proc. natl. Acad. Sci. U.S.A., 47, 390-396.

FRANKEL, R. 1956. Graft induced transmission to progeny of cytoplasmic male sterility in Petunia. Science, 124, 684-685.

FRANKEL, R. 1962. Further evidence on graft induced transmission to progeny of cytoplasmic male sterility in Petunia. Genetics, 47, 641-646.

FRANKEL, R. 1963. Alternative cytoplasmic and nuclear hereditary elements in Petunia. Proc. XI Int. Congr. Genetics, 1, 12, 14.

frankel, R. 1964. The nature of cytoplasmic male sterility in Petunia. Spec. Bull. Nat. Univ. Inst. Agric., Rehovot, No. 69 (Hebrew, with English summary).

FRANKEL, R. 1965. Transfer of cytoplasmic male sterility between graft components in Petunia. Israel F. Agr. Res., 15, 105 (Abstract).

FRANKEL, R. 1967. Alternative cytoplasmic and chromosomal hereditary elements in male sterile Petunia. Israel F. Bot., 16, 158 (Abstract).

HAYES ,W. 1969. In Bacterial Episomes and Plasmids. Ciba Foundation Symposium. London: J. A. Churchill Ltd. 
JacoB, P., AND Wollman, E. L. 1961. Sexuality and Genetics of Bacteria. New York: Academic Press Inc.

JAIN, s. x. 1959. Male sterility in flowering plants. Bibliog. Genet., 18, 101-166.

LACADENA, L. R. 1968. Hybrid wheat. VII. Test on the transmission of cytoplasmic male sterility in wheat by embryo-endosperm grafting. Euphytica, 17, 439-444.

MICHAELIS, P. 1960. Uber reziprok verschiedene Sippenbastarde by Epilobium hirsutum.

II. Uber die Konstanz des Plasmons der Sippe Jena. Z. Vererbungslehre, 78, 223-237. MICHAELus, P. 1964. Virus und Zytoplasmatische Vererbung. Z. Pfanzenzücht., 52, 333-354. OHrA, y. 1961. Grafting and cytoplasmic male sterility in Capsicum. Seiken Ziko, 12, 35-43. RHOADES, M. M. 1950. Gene induced mutation of a heritable cytoplasmic factor producing male sterility in maize. Proc. natl Acad. Sci. U.S.A., 36, 634-365.

SAND, s. A. 1960. Autonomy of cytoplasmic male sterility on grafted scions of tobacco. Science, 131, 665 . 